\title{
Ultrasound Guided Erector Spinae Plane Catheter Versus Video-Assisted Paravertebral Catheter Placement in Minimally Invasive Thoracic Surgery: Comparing Continuous Infusion Analgesic
} Techniques on Early Quality of Recovery, Respiratory Function and Chronic Persistent Surgical Pain: Study Protocol for A Double-Blinded Randomised Controlled Trial.

Aneurin Moorthy ( $\nabla$ aneurin.moorthy@gmail.com )

Mater Misericordiae University Hospital https://orcid.org/0000-0001-7325-8908

Aisling Ni Eochagain

St James's University Hospital

\section{Eamon Dempsey}

St James's University Hospital

Donal Buggy

Mater Misericordiae University Hospital

Research Article

Keywords: Erector Spinae Catheter, Paravertebral Catheter, Minimal Invasive Thoracic Surgery, Quality of Recovery, Chronic Persistent Surgical pain.

Posted Date: July 14th, 2021

DOI: https://doi.org/10.21203/rs.3.rs-703831/v1

License: (a) (1) This work is licensed under a Creative Commons Attribution 4.0 International License. Read Full License

Version of Record: A version of this preprint was published at Trials on December 1st, 2021. See the published version at https://doi.org/10.1186/s13063-021-05863-9. 


\section{Abstract}

Background: Compared to conventional thoracotomy, minimally invasive thoracic surgery (MITS) can reduce postoperative pain, reduce tissue trauma and contribute to better recovery. However, it still causes significant acute post-operative pain. Truncal regional anaesthesia techniques such as paravertebral and Erector Spinae blocks have shown to contribute to post-operative analgesia after MITS. Satisfactory placement of an ultrasound guided thoracic paravertebral catheter can be technically challenging compared to ultrasound guided Erector Spinae catheter. However, in MITS an opportunity arises for directly visualised placement of a paravertebral catheter by the surgeon under thoracoscopic guidance. Alongside with thoracic epidural, paravertebral block is considered the "gold standard" of thoracic regional analgesic techniques. To best of our knowledge, there are no randomised controlled trials comparing Surgeon-administered Paravertebral catheter and Anaesthesiologist-assisted Erector Spinae catheter for MITS in terms of patient centred outcomes such as quality of recovery.

Methods: This trial will be a prospective, double-blinded randomised controlled trial. A total of 80 eligible patients will be randomly assigned to receive either an Anaesthesiologist-assisted ultrasound guided Erector Spinae catheter or Surgeon-assisted video-assisted Paravertebral catheter, in a 1:1 ratio following induction of general anaesthesia for minimally assisted thoracic surgery. Both groups will receive the same standardized analgesia protocol for both intra and postoperative periods. The primary outcome is defined as Quality of Recovery (QoR-15) score between the two groups at 24 \& 48 hours postoperative. Secondary outcomes include assessment of chronic persistent surgical pain (CPSP) at 3 months postoperative using Brief Pain Inventory (BPI) Short Form and Short Form McGill (SF-15) questionnaires, assessment of post-operative pulmonary function, area Under the Curve for Verbal Rating Score for pain at rest and on deep inspiration versus time over 48 hours, total opioid consumption over 48 hours and post-operative complications and morbidity as measured by the Comprehensive Complication Index.

Discussion: Despite surgical advancements in thoracic surgery, severe acute post-operative pain following MITS is still prevailing. This study will provide recommendations about the efficacy of anaesthesia-administered ultrasound guided Erector Spinae catheter or surgeon-administered, video assisted paravertebral catheter techniques for early quality of recovery following MITS.

Trial registration: This trial was pre-registered on ClinicalTrials.gov Identifier: NCT04729712. Registered on 28 January 2021. All item from the World Health Organisation Trial Regsitration Data set have been included. https://clinicaltrials.gov/ct2/show/NCT04729712

\section{Administrative Information}


Title $\{1\} \quad$ Ultrasound guided erector spinae plane (ESP) catheter versus video-assisted paravertebral catheter placement in minimally invasive thoracic surgery (MITS): Comparing continuous infusion analgesic techniques on early quality of recovery, respiratory function and chronic persistent surgical pain: study protocol for a doubleblinded randomised controlled trial.

Trial registration

$\{2 \mathrm{a}$ and

2b\}.

Protocol

version $\{3\}$

Funding $\{4\}$

Author

details $\{5 a\}$
This trial was pre-registered on ClinicalTrials.gov Identifier: NCT04729712. Registered on 28 January 2021. All item from the World Health Organisation Trial Regsitration Data set have been included. https://clinicaltrials.gov/ct2/show/NCT04729712

Protocol version 8: 01 May, 2021

This trial is being funded internally from the division of Anaesthesiology \& Perioperative Medicine, Mater University Hospital. The trial has also received external funding (total of 1,000 euros) from the Irish Society of Regional Anaestheia (ISRA).

(1): Dr Aneurin Moorthy*: Anaesthesia research fellow, Division of Anaesthesiology \& Perioperative Medicine, Mater University Hospital, Dublin, Ireland; aneurin.moorthy@gmail.com *Correspondence

(2): Dr Aisling Ni Eochagain: Anaesthesia Specialist Registrar, Department of anaesthesia and critical care, St James's University Hospital, Dublin, Ireland; aislingnie@gmail.com.

(4): Dr Eamon Dempsey: Anaesthesia Specialist Registrar, Department of anaesthesia and critical care, St James's University Hospital, Dublin, Ireland; edempsey376@msn.com

(3): Professor Donal J. Buggy**: Consultant Anaesthesiologist, Division of Anaesthesiology \& Perioperative Medicine, Division of Anaesthesiology \& Perioperative Medicine, Mater University Hospital, Dublin, Ireland; donal.buggy@ucd.ie **Principal investigator

Name and contact information for the trial sponsor $\{5 b\}$

Role of sponsor $\{5 c\}$

Division of Anaesthesiology \& Perioperative Medicine, Division of Anaesthesiology \& Perioperative Medicine, Mater University Hospital, Dublin, Ireland. Anaes@mater.ie office: Office: 003531803 2286/2281

\section{Introduction}

\section{Background and rationale $\{6 a\}$}

Minimally invasive thoracic surgery (MITS) is a surgical method used to perform lung surgery through small incisions between the ribs and includes both Video-Assisted Thoracic Surgery (VATS) and Robotic Assisted Thoracic Surgery (RATS) ${ }^{1}$. MITS has increased to almost half of all thoracic surgery in the past decade $^{2}$. Compared to conventional thoracotomy, MITS can reduce postoperative pain, reduce tissue 
trauma and contribute to better recovery ${ }^{3,4}$. However, it still causes severe acute post-operative pain ${ }^{3}$ and is a risk factor for developing Chronic Post-Surgical Pain (CPSP) ${ }^{5}$.

Erector spinae plane (ESP) block has emerged as a new regional anaesthesia technique which has had promising early results in attenuating this severe acute pain of MITS. In a recent RCT among MITS patients, single shot ESP block improved QoR-15 and reduced overall complications at $24 \mathrm{hr}$ compared with single-shot serratus anterior plane block ${ }^{6}$.

Paravertebral block (PVB) has been widely used for analgesia after thoracic surgery for over two decades, because it reduces postoperative pain and opioid requirements compared with systemic analgesia ${ }^{7,8}$ and alongside epidural analgesia, is considered the "gold standard" of thoracic regional analgesic techniques ${ }^{9}$.

Both ESP and PVB have usually been described as a single shot technique. This has the advantage of convenience but is limited by the finite duration of analgesia, extending to no more than 12 hour at maximum and often considerably less ${ }^{6,10}$. Catheter techniques offer the prospect of flexibility and prolonged analgesia.

Placement of a thoracic paravertebral catheter can be achieved by an anatomical landmark or ultrasound technique ${ }^{11}$. Ultrasound technique has a higher success rate and safer profile when compared to the landmark technique ${ }^{12}$ but is technically challenging. Moreover, despite correct detection of the paravertebral space by ultrasound, the final location of paravertebral catheters can be unknown or problematic and cadaveric studies have revealed that catheters have been misplaced in up to $40 \%$ of placements $^{13,14}$.

However, in MITS an opportunity arises for directly visualised placement of a paravertebral catheter by the surgeon under thoracoscopic guidance ${ }^{15}$. In a meta-analysis ${ }^{16}$ comparing paravertebral to epidural analgesia, lower failure rates in paravertebral placement was attributed to the practice of intra-operative surgical placement of the catheter under direct vison. Therefore, insertion of the catheter and verification by the surgeon under direct vision could be a more reliable and safe method for correct placement of a paravertebral catheter, but there are no randomised controlled trials examining the efficacy of this technique.

In addition, there are limited clinical effectiveness trials on the catheter-based ESP analgesia technique using patient-centred outcomes. Further, no study has evaluated the effect of acute analgesia over 24-48 hours with continuous regional nerve block on chronic persistent surgical pain (CPSP) at 3 months after MITS.

\section{Objectives $\{7\}$}

Our main aim and corresponding hypotheses are outlined below. 
We will conduct a randomised controlled clinical trial comparing the efficacy of anaesthesiologistadministered, ultrasound guided ESP catheter analgesia to surgeon-administered, video-assisted PVB catheter analgesia. We will test the hypothesis that anaesthesiologist-administered ultrasound guided ESP catheter analgesia is equivalent to surgeon-administered, video-assisted PVB catheter analgesia, in terms of early Quality of Recovery (QoR-15) and post-operative respiratory spirometric function at 24-48 hours, and CPSP at 3 months after MITS surgery.

\section{Trial design $\{8\}$}

This manuscript outlines a research protocol for a double-blind, randomised controlled clinical trial. Patients that volunteer to participate in this clinical trial will be equally $(1: 1)$ and randomly allocated into one of two intervention groups: anaesthesiologist-administered ultrasound guided Erector Spinae catheter group (ESP catheter group) or surgeon-administered, video-assisted Paravertebral Block catheter group (PVB group). Figure.1 illustrates the study flow chart. Recruitment commenced on 12 May 2021, and it is expected to take between 9 and 12 months for completion.

\section{Methods: Participants, Interventions And Outcomes Study setting $\{9\}$}

The authors aim to conduct a multicentre trial. Ethical approval has been granted for Mater Misericordiae University Hospital (MMUH), Dublin, Ireland and recruitment has begun. We are currently assessing suitability and seeking ethical approval for two other potential clinical sites in Dublin, Ireland.

\section{Eligibility criteria $\{10\}$}

The eligibility criteria for patients to enroll in this study are as follows:

Inclusion criteria:

1. ASA grade 1 to 4 patients

2. Male and female age $18-85 \mathrm{yr}$

3. Unilateral MITS (VATS and RATS).

Exclusion criteria:

1. Absence of written consent

2. Unexpected conversion of MITS to open thoracotomy

3. Contraindications to peripheral regional anaesthesia block:

Infection at local site

Allergy to local anaesthesia medications

Patient refusal

Previous or existing neurological deficit 
Anticoagulated patients

Infection at local site

Known Dementia at time of MITS and inability to give informed consent

4. Unexpected postoperative admission to ICU for continued ventilation

5. Existing chronic pain condition

6. History of opioid abuse

\section{Who will take informed consent? \{26a\}}

Potential participants for this trial will be identified by a member of the surgical, anaesthetic or research team. A member of the research team will analyse these patient's electronic medical records to determine if they are potential candidates for this trial, i.e., if they meet the inclusion criteria and have no exclusions.

The suitable patient will be approached the evening before surgery if available. Alternatively, patients will be approached on the ward on the morning of surgery and their suitability to participate in the trial will be confirmed. The purpose of the trial, peripheral nerve blocks (including benefits and risks) and method of follow up will be explained to the patient. A comprehensive and informative leaflet will be given to each patient and they will be afforded an adequate amount of time (minimum 10 mins) to study it.

Participants will be informed that their participation in the study is entirely voluntary and they will have the opportunity to withdraw from the study at any time and this will not affect the quality of care they receive. Subsequently, a member of the research team will obtain informed written consent from the participant.

\section{Additional consent provisions for collection and use of participant data and biological specimens $\{26 \mathrm{~b}\}$}

Not applicable. No data and biological specimens will be collected for use in ancillary studies.

\section{Interventions}

\section{Explanation for the choice of comparators $\{6 b\}$}

Erector Spinae block has been embraced by many anaesthesiologists, however, to the best of our knowledge there are no clinical effectiveness trial comparing anaesthesiologist-administered, ultrasound guided Erector Spinae catheter to Surgeon-administered, video-assisted Paravertebral Block catheter techniques for minimal invasive thoracic surgery in terms of patient centred outcomes and therefore this clinical trial is warranted.

\section{Intervention description $\{11$ a $\}$}

\section{ESP catheter group: Anaesthesiologist-administered, ultrasound guided Erector Spinae catheter [n = 45]}


This peripheral nerve block will be performed or supervised by a consultant anaesthesiologist in regional anaesthesia with experience in placement of ESP catheter. The block will be performed as follows: the patient will be positioned in the lateral decubitus position and the skin will be cleaned with chlorhexidine gluconate $0.5 \%$ / isopropyl alcohol $70 \%$ solution (ChloraPrep; Becton Dickinson, New Jersey, NJ, USA). A linear ultrasound transducer (SonoSite HFL 50x; SonoSite Inc.) will be placed in a sterile cover. This ultrasound probe will be used to identify the $T 5$ spinous process and the erector spinae muscle group superficial to it. An 18G Tuohy Epidural needle (B. Braun medical) will then be advanced in a cranialcaudal direction. The needle tip will be slowly advanced under ultrasound guidance (In-plane technique) until it is located within the interfascial plane deep to the erector spinae muscle group and superior to the transverse process. Once in position, $10 \mathrm{ml}$ of $0.9 \%$ normal saline will be injected to confirm satisfactory needle position. Following this, $20 \mathrm{mls}$ 0.375\% Leveobupivacaine will be injected and then a $20 \mathrm{G}$ Nylon Epidural catheter (Perifix Epidural Catheter, B. Braun medical) will be advanced into this interfascial plane. The catheter will then be attached to a luer lock connector with an antibacterial filter and secured to the patients back using surgical steri strips and standard dressings. A further bolus of $10 \mathrm{mls} 0.25 \%$ Levobupivacaine will be administered via the catheter towards the end of the surgical procedure ( $>1$ hour since last dose).

\section{PVB catheter group: Surgeon-administered, video-assisted Paravertebral Block catheter. [n = 45]}

This nerve block will be performed or supervised by a consultant thoracic surgeon with experience in placement of paravertebral catheter under thoracoscopic guidance. The paravertebral block will be conducted at the start of the surgery after the thoracoscopic ports have been inserted. The block will be performed as follows: A percutaneous puncture point, which is equidistant to the upper and lower intercostal space, $2-3 \mathrm{~cm}$ lateral to the midline at the level between T4-T5 will be marked. An $18 \mathrm{G}$ Tuohy Epidural needle (B. Braun medical) will be advanced perpendicularly through the chest wall at this marked point to lie within the paravertebral space. The thoracoscope will be used to ensure that the epidural needle does not puncture the pleura and then $10-20 \mathrm{ml}$ of $0.9 \%$ normal saline will be injected through the epidural needle to confirm satisfactory position of the needle. This will appear as an induration under the pleura which can be easily seen thoracoscopically. A 20 G Nylon Epidural catheter (Perifix Epidural Catheter, B. Braun medical) will then be advanced into the paravertebral space through the epidural needle. Again, the thoracoscope will be used to ensure that the epidural catheter does not puncture the pleura and lies within the paravertebral space. The catheter will then be attached to a luer lock connector with an antibacterial filter and secured to the patients back using surgical steri strips and standard dressings. A bolus of $20 \mathrm{ml} 0.375 \%$ levobupivacaine will then be injected into the paravertebral space by using the catheter.

A further bolus of $10 \mathrm{mls} 0.25 \%$ Levobupivacaine will be administered via the catheter towards the end of the surgical procedure ( $>1$ hour since last dose) 


\section{Criteria for discontinuing or modifying allocated interventions $\{11 \mathrm{~b}\}$}

Criteria for discontinuing study protocol are as follows:

1. Unexpected conversion of MITS to open thoracotomy

2. Unexpected regional anaesthesia complication

3. Suspected or confirm diagnosis of local anaesthesia toxicity

4. Unexpected postoperative admission to intensive care unit

5. Inability to place ESP or PVB catheter

6. Patient request to be withdrawn from study

7. Clinical concern of patient's care from surgical, anaesthesia or research team.

\section{Strategies to improve adherence to interventions $\{11 \mathrm{c}\}$}

A study flow chart (Fig. 1) and analgesia \& anti-emetic protocol (Fig. 2) will be made available in the operating theatre, Surgeons \& Anaesthesiologist performing the intervention, Post Anaesthesia Care Unit (PACU) department and data collectors for the purpose of protocol adherence.

\section{Relevant concomitant care permitted or prohibited during the trial $\{11 \mathrm{~d}\}$}

All patients will undergo general anaesthesia (GA) as part of standard of care for their video-assisted thoracic surgery. Intravenous induction of GA will be conducted or supervised by a consultant anaesthesiologist. Induction of GA will be achieved by administrating intravenous Fentanyl, Propofol and a neuromuscular blockade agent at the discretion of the anaesthesiologist. An analgesia and anti-emetic concomitant care plan will be standardised for the pre-surgical, intra-operative, PACU and Post-operative period. This is outlined in Fig. 2.

\section{Provisions for post-trial care $\{30\}$}

Patients that are enrolled into the study are covered by indemnity for negligent harm, through the standard HSE (Health Service Executive) indemnity arrangements. If any participant suffers from any complications arising directly from either intervention, he/she will receive standard post-operative management which may include from the surgical team, pain medicine department and allied health care professions. In addition, all investigators in all potential clinical sites will be respective employees of the hospital and covered by the clinical indemnity scheme.

\section{Outcomes $\{12\}$}

Primary Outcome Measures:

1. Quality of Recovery (QoR-15) score between Anaesthesiologist-administered ultrasound guided Erector Spinae block and Surgeon-administered video-assisted Paravertebral block at 24 \& 48 hours 
postoperative. [ Time Frame: $24 \& 48$ hour postoperative].

QoR-15 is a 15-parameter questionnaire which has been recommended as an optimum tool to evaluate overall patient recovery after surgery and this includes postoperative pain. Participants will complete this questionnaire at 24 hours after their surgery. It is scored between 0 and 150, where 150 indicates that the patient has had an excellent recovery.

Secondary Outcome Measures:

1. Chronic Persistent Surgical Pain (CPSP) after Minimally Invasive Thoracic Surgery (MITS) [ Time Frame: 3 months postoperative].

This will be achieved by assessing the patient for chronic pain by using Brief Pain Inventory (BPI) Short Form and Short Form McGill (SF-15) questionnaires. This assessment will be conducted by phone call by a member of the research team.

1. BPI assess for quality of life and pain and its scale is measured between $0-10$, where ' 0 ' indicates no interference with quality of life and no pain and ' 10 ' indicates severe interference with quality of life and severe pain.

SF-15 assess for neuropathic pain and its scale is measured between $0-100$, where ' 0 ' indicates no neuropathic pain and ' 100 ' indicates severe neuropathic pain.

$\mathrm{BPI}$ assess for quality of life and pain and its scale is measured between $0-10$, where ' 0 ' indicates no interference with quality of life and no pain and ' 10 ' indicates severe interference with quality of life and severe pain.

Pulmonary Function Assessment [ Time Frame: Pre-operatively day 0, post-operative day 1 \& postoperative day 2 ]

Pulmonary Function Assessment [ Time Frame: Pre-operatively day 0, post-operative day 1 \& postoperative day 2 ]

Pulmonary function assessment will be evaluated pre-operatively (day 0 ) before induction of GA and at post-operative day 1 and 2 using a bedside incentive spirometry. An average of three maximum inspiration volumes will be measured with the patient in the sitting position. A change in maximum inspiratory volumes between pre-operative and postoperative day 1 and 2 will be assessed.

Pulmonary function assessment will be evaluated pre-operatively (day 0 ) before induction of GA and at post-operative day 1 and 2 using a bedside incentive spirometry. An average of three maximum inspiration volumes will be measured with the patient in the sitting position. A change in maximum inspiratory volumes between pre-operative and postoperative day 1 and 2 will be assessed.

Area Under the Curve (AUC) of Verbal Rating Score (VRS) for pain at rest and on deep inspiration versus time over 48 hours [ Time Frame: 60-120 mins in PACU, 24 \& 48 hour post-operative ] 
Area Under the Curve (AUC) of Verbal Rating Score (VRS) for pain at rest and on deep inspiration versus time over 48 hours [ Time Frame: 60-120 mins in PACU, $24 \& 48$ hour post-operative ]

Verbal Rating scale is measured from $0-10$, where ' 0 ' indicates no pain and ' 10 ' indicates severe pain.

Verbal Rating scale is measured from $0-10$, where ' 0 ' indicates no pain and ' 10 ' indicates severe pain.

Time taken to site ESP and PVB cather [ Time Frame: Intra-operative ]

1. Time of administration of first intravenous opioid

2. Total 24 and 48 hour opioid consumption

3. Documentation of adverse events

This includes intra-operative haemodynamic changes, post-operative hypotension, nausea \& vomiting, pruritis, block failure and block related complications.

This includes intra-operative haemodynamic changes, post-operative hypotension, nausea \& vomiting, pruritis, block failure and block related complications.

Length of hospital stay [ Time Frame: 1 month]

4. Comprehensive Complication Index Calculator (CCl) [ Time Frame: 1 month ]

The $\mathrm{CCl}$ is calculated as the sum of all complications that are weighted for their severity. The final formula yields a continuous scale to rank the severity of any combination of complications from 0 to 100 in a single patient.

\section{Participant timeline $\{13\}$}

The schedule of enrolment, interventions and assessments is outlined in Fig. 3.

\section{Sample size $\{14\}$}

Data will be recorded in Excel (Microsoft ${ }^{\mathrm{TM}}$ ) and imported into GraphPad Prism v8 for analysis. All data will be stored according to EU Directive 2019 on General Data Protection Regulations. Data will be inspected and tested for distribution according to the Kolmogorov-Smirnov test. Normally distributed data will be compared between study arms using the unpaired $t$ test, whereas non-normally distributed data will be compared using Mann-Whitney $U$ test. All data will be summarised as mean \pm SD or median (25$75 \%$ range) as appropriate.

Data will be analysed by GraphPad Prism version 8 (GraphPad, Salt Lake City, UT, USA). The primary outcome will be the QoR-15 score at 24 and 48 hours post operatively. The established minimum clinically important difference in QoR-15 is $8.0^{17}$ and the SD of QoR-15 scores is typically between 10- 
16. [range of QoR score is 1-150]. We have chosen a SD of 12 to reflect our study population. Therefore, assuming Type I error $=0.05$ and Type II error $=0.2$ ( $80 \%$ power to detect this difference), then $n=36$ patients will be required in each group. We aim to enroll $n=40$ each group to allow for loss to follow up, missing data or withdrawal of consent.

\section{Recruitment $\{15\}$}

Members of the anaesthestic, surgical and research team from the clinical site will participate in the recruitment process.

\section{Assignment of interventions: allocation}

\section{Sequence generation $\{16 \mathrm{a}\}$}

Patients will be randomised to either ESP or PVB group by using an online computer-generated block randomisation. Block randomisation will occur in groups of 10 to ensure even numbers of participants in each arm of the study. The investigators for this trial will not have access to the randomisation key/seed until the study has been completed.

\section{Concealment mechanism $\{16 \mathrm{~b}\}$}

The patient study number and group allocation will be typed onto separate pages and concealed in sequentially numbered, opaque, sealed envelopes. The randomisation process will be performed by an independent third party that is not involved conducting this trial.

\section{Implementation $\{16 \mathrm{c}\}$}

After confirming the informed consent form for participating in this trial has been signed by the participant, the sealed envelope will be opened by the treating anaesthesiologist to reveal the group allocation. This process will occur after induction of general anaesthesia to ensure the patient is blinded to the study intervention.

\section{Assignment of interventions: Blinding Who will be blinded $\{17 \mathrm{a}\}$}

This study will be a double-blinded clinical trial. Patients will be blinded to the study because they will receive the intervention after they have been put under GA. Members of the research team involved in the data collection and analysing the data will be masked to group allocation. The treating anaesthesiologist and surgical team will not be blinded

\section{Procedure for unblinding if needed $\{17 \mathrm{~b}\}$}

A participant's allocations will be revealed immediately if there was a clinical concern, i.e., If patient met criteria for discontinuing study protocol (part 11b).

Data collection and management 


\section{Plans for assessment and collection of outcomes $\{18 \mathrm{a}\}$}

Data collection will occur at three time points (Pre-operative, Intra-operative and post-operative). Data will be derived from a combination of electronic \& paper patient records and directly from the patient by means of completing a questionnaire. In addition, further post-operative data (assessing for CPSP) will be collected by phone call after the patient has been discharged. The same member of the research team (blinded to the intervention) will collect all peri-operative data.

\section{Plans to promote participant retention and complete follow- up $\{18 b\}$}

Trial participants will receive an extensive patient information leaflet (PIL) about the study. A member of the research team will explain this PIL, study set-up and the study interventions. The three-month follow up call for assessment of CPSP will be explained to the patient during the consent process. The importance of completion of this follow up will be stressed.

\section{Data management $\{19\}$}

All patient data collected will be handled in accordance with European Union General Data Protection Regulations (EU 2016/679). Data will be initially collected manually and then transcribed onto Microsoft Excel. Data collected from the clinical site will be stored securely in the Department of Anaesthesiology at the site hospital, on a password-protected desktop computer stored in a locked office, such that only investigators assigned to data input, processing and analysis will have access. Data will be collected directly from source documents into the de-identified encoded paper Case Record Form (CRF) and subsequently entered into the electronic CRF. A copy of the original hardcopy CRF will be stored within a locked cabinet/office accessible to authorised personnel only in accordance with local and national regulations.

\section{Confidentiality \{27\}}

All research data will be stored using a study identification number for each patient. An identifiable patient data page reporting the assigned patient identification code will be stored separately also in a locked cabinet/office (accessible to authorised personnel only) in order to record in-hospital outcomes, post-operative 3-month follow up, supply missing data points, and to allow potential monitoring visits by National Coordinating Investigators. This data page will only be made available to members of the research team responsible for data input and the principal investigator. No patient identification details will be reported in any future publications.

Plans for collection, laboratory evaluation and storage of biological specimens for genetic or molecular analysis in this trial/future use $\{33\}$

Not applicable, no samples will be collected.

\section{Statistical methods}




\section{Statistical methods for primary and secondary outcomes $\{20$ a $\}$}

The collected raw data will be initially inspected for any errors, this includes but is not limited to double entry errors, missing data and data that was incorrectly entered. The data will be tested for normal distribution according to the Shapiro-Wilk test. Normally distributed data will be compared between the two groups using the unpaired student $t$ test and non-normal distributed data will be compared by using Mann-Whitney $\mathrm{U}$ test. All data will be summarised as mean $\pm \mathrm{SD}$ and $\mathrm{p}$ value $<0.05$ will be considered statistically significant.

\section{Interim analyses $\{21 \mathrm{~b}\}$}

There are no interim analyses planned.

\section{Methods for additional analyses (e.g. subgroup analyses) $\{20 \mathrm{~b}\}$}

There are no subgroup analyses planned.

Methods in analysis to handle protocol non-adherence and any statistical methods to handle missing data $\{20 \mathrm{c}\}$

The primary outcome will be assessed using an unpaired student t test analysis. Or Mann-Whitney U test, depending on data distribution. Given our expectation that very few patients will be lost to follow-up during their inpatient stay and protocol adherence strategies as mentioned above, we expect missing data will be reduced to a minimum when analysing the primary outcome. If statistical method is needed to account for missing data in the secondary outcomes (e.g., CPSP survey at 3-month follow up), multiple imputation will be used.

\section{Plans to give access to the full protocol, participant level- data and statistical code $\{31 \mathrm{c}\}$}

The collated data collected by the investigators will be retained for a maximum 5 years after analysis has been completed. We will deliver a completely de-identified data set an appropriate data upon reasonable request and in agreement with the principal investigator and data protection officer.

\section{Oversight and monitoring}

\section{Composition of the coordinating centre and trial steering committee $\{5 d\}$}

The Trial steering committee will meet monthly to evaluate progress, address ongoing organisational and logistical issues and consider any adverse effects. There will be a research leader for the clinical site of whom will provide monthly reports to the principal investigator. 


\section{Composition of the data monitoring committee, its role and reporting structure $\{21 \mathrm{a}\}$}

A data monitoring committee (DMC) has not been appointed for this study. A data protection impact assessment (DPIA) screening tool was completed, and it was analysed by the hospital's data protection officer (DPO). In agreement with the DPO, this study poses a low risk to the rights and freedoms of natural persons and therefore a formal DPIA was not needed. Moreover, due to the rapid expected inclusion of participants to this trial, data collection is expected to be completed in less than nine months and known minimal inherited risks associated with this trial, a DMC was not appointed.

\section{Adverse event reporting and harms $\{22\}$}

Any unexpected complications that may arise from this trial will be documented and reported to the principal investigator, surgical consultant and to the relevant hospital patient safety board.

\section{Frequency and plans for auditing trial conduct $\{23\}$}

For the purpose of this trial, an initial auding process maybe conducted using a risk-based approach. This would initially involve focusing on a center which may have the largest number of enrolment and/or lost of follow-up rates. The auditing process would include exploring datasets and analysing for accuracy, missing data, duplicate data and adhering to data protection guidelines. This process would be conducted by an independent reviewer who has no involvement with the current trial (e.g., research nurse that is affiliated with the clinical site but is not involved with the current trial).

A research nurse affiliated with $\mathrm{MMUH}$ anaesthesiology but not involved in this trial may undertake an audit involving exploring datasets from this and the other institution. This would be precipitated by risk indices including high rates of enrolment and drop-out rates.

\section{Plans for communicating important protocol amendments to relevant parties (e.g. trial participants, ethical committees) $\{25\}$}

We define a substantial modification of the study protocol as changes which may affect the outcome of the study or patient safety. Changes include: any modification to the aims of the study, study design, the inclusion or exclusion criteria or any alternations of the study interventions (using new procedural equipment or conducting intervention which deviate original description). Any amendment will be agreed upon the principal investigator of this trial and will seek approval by the Ethics Committee/IRB. Minor changes of the protocol include any administrative changes or alternation of the analgesia plan that do not impact patient safety or the conduct of the trial (e.g., changes to anti-emetic medications). The Ethics Committee/IRB may be notified of minor changes at the discretion of the principal investigator.

Dissemination plans $\{31 \mathrm{a}\}$ 
The results from this clinical trial will be fully disclosed by means of publication in an international peerreviewed journal and by oral/poster presentations at national \& international scientific meetings. Both positive and negative results will be reported.

\section{Discussion}

In conducting this randomised control trial we aim to investigate the efficacy, in terms of quality of recovery, of either an Anaesthesiologist-assisted ultrasound guided Erector Spinae Plane catheter or Surgeon-assisted video-assisted Paravertebral catheter, following induction of general anaesthesia for minimally assisted thoracic surgery. Alongside epidurals, paravertebral blocks have been widely used for analgesia after thoracic surgery for many years and are considered the "gold standard" of thoracic regional analgesic techniques. ${ }^{9}$ Surgical advancements in the form of MITS, results in less tissue trauma and less postoperative pain for patients undergoing thoracic surgery and so the use of less invasive regional techniques may be more appropriate for this subgroup of thoracic patients. ${ }^{3,4}$ The ESP block has become progressively more popular in recent times, and its use has been reported in a range of thoracic, spinal and abdominal surgeries. ${ }^{18-21}$ Single shot truncal regional anaesthesia techniques such as paravertebral and ESP blocks have shown to contribute to post-operative analgesia after MITS but they are restricted by the limited duration of analgesia, which extends to no more than 12 hours in duration. 6,10 Catheter techniques offer opportunities of increased flexibility and prolonged analgesia. The use of catheter techniques for MITS are not well documented and existing pilot feasibility trials have used the traditional end points of opioid consumption and pain scores to assess its efficacy. ${ }^{22}$ To the best of our knowledge, no trial to date has examined the impact ESP catheters on quality of recovery in MITS patients.

Current evidence suggests that the implementation of Enhanced Recovery After Surgery (ERAS) programmes in thoracic surgery may lead to improvements in functional recovery, length of stay, opioid use, complications and readmissions. ${ }^{23}$ Because of their estimated analgesic advantages, fascial plane catheters may have a role as part of an ERAS programme and merit further investigation.

While MITS surgery is associated with less tissue trauma and lower pain scores than open thoracic surgery there has been a heavy reliance on the use of opioids for management of acute pain in the postoperative period. ${ }^{24}$ Pre-emptive administration of these agents is no longer recommended. ${ }^{25}$ Opioid use combined with poorly controlled pain scores postoperatively have been identified as risk factors associated with opioid misuse during the postoperative period. ${ }^{26}$ Due to current public health concerns regarding addiction to prescription opioids administered postoperatively, that there is a real need to direct our focus towards non-opioid based multimodal analgesic strategies such as fascial plane blocks.

This protocol will result in the anaesthesiologist and surgeon not being blinded to the group allocation as they will be required to perform the block. However, the primary outcome is the QoR-15 score at $24 \mathrm{hr}$ postoperatively which will be measured by researchers blinded to the group allocation. We have chosen to exclude patients with existing chronic pain conditions or with a history of opioid abuse. The justification for this decision comes from the fact that these patients are more likely to develop 
postoperative chronic pain and so the results of our three month chronic pain scores may be affected by the inclusion of these patients. Pain scores and quality of recovery will be recorded up to $48 \mathrm{~h}$ postoperatively. We acknowledge that for some patients having MITS their recovery will continue beyond 48 hours, however the acute postoperative pain following MITS is reduced by Day 3 and many of our patients are discharged home at this stage. Length of stay and postoperative complications will continue to be observed after this timeframe and patients will be followed up until 3 months postoperatively. Paravertebral and ESP catheters will be placed under general anaesthesia, therefore formal dermatomal assessment of block function will not be undertaken. Therefore we will not be formally testing block effectiveness. However, the practice of placing these catheters under ultrasound guidance or thorascopic vision after induction of general anaesthesia is in line with common clinical practice, and therefore our findings should still be applicable to widespread clinical practice. Patients' preoperative QoR-15 will not be assessed in this study. Therefore, we will not have a baseline from which to compare postoperative QoR-15 scores. However, QoR-15 was designed specifically for postoperative use, and we will apply this scoring tool to both randomised cohorts equally. Moreover, the ability of QoR-15 in the immediate preoperative period to give an accurate baseline has been questioned. ${ }^{27}$ By focusing on patient-centred outcomes in our trial design, we hope to elucidate whether these relatively straightforward fascial plane blocks can enhance recovery and improve safety for patients by reducing postoperative morbidity and complications.

\section{Trial Status}

The trial is registered on ClinicalTrials.gov Identifier: NCT04729712. The current protocol is version 8 of $01 / 05 / 2021$. Participant recruitment began on $12 / 05 / 2021$ and full patient recruitment is estimated to be completed by July 2022 .

\section{Abbreviations}

MITS: Minimally Invasive Thoracic Surgery

QoR-15: Quality of Recovery

CPSP: Chronic Persistent Surgical Pain

BPI: Brief Pain Inventory

SF-15: Short Form McGill

VATS: Video-Assisted Thoracic Surgery

RATS: Robotic Assisted Thoracic Surgery

ESP: Erector Spinae Plane 
RCT: Randomised Control Trial

PVB: Paravertebral Block

MMUH: Mater Misericordiae University Hospital

SJH: St James University Hospital

ASA: American Society of Anaesthesiology

ICU: Intensive Care Unit

PACU: Post Anaesthesia Care Unit

GA: General Anaesthesia

HSE: Health Service Executive

AUC: Area Under the Curve

CCl: Comprehensive Complication Index Calculator

SD: Standard Deviation

PIL: Patient information Leaflet

CRF: Case Record Form

DMC: Data Monitoring Committee

DPIA: Data Protection Impact Assessment

DPO: Data Protection Officer

\section{Declarations}

\section{Acknowledgements}

We would like to thank all patients for their time and willingness to participate in this clinical trial. In addition, we thank our surgical colleagues; Dr Karen Redmond, Dr Dona Eaton and Dr David Healy for their commitment and help in implementing the study interventions involved in this trial; our anaesthesia colleagues for their support to date and our nursing colleagues in theatre \& PACU for their kind care for our patients.

Authors' contributions \{31b\} 
AM \& DJB involved in trial coordination, trial concept, design, proposal and protocol development. AM involved in data collection. AE involved in trial coordination, acquiring data, proposal development. DJB is the principal investigator. $A M, A E, E D \& D J B$ contributed equally to the preparation of this manuscript. All authors read and approved the final manuscript.

\section{Funding $\{4\}$}

This trial is being funded internally from the Division of Anaesthesiology \& Perioperative Medicine, Mater University Hospital, Dublin, Ireland and an external grant was received from the Irish Society of Regional Anaesthesia (ISRA). Both parties have no role in the design of the study, data collection and interpretation of data or in the wiring of the manuscript.

\section{Availability of data and materials $\{29\}$}

The final trial dataset will be made available from the corresponding and principal author upon reasonable request.

\section{Ethics approval and consent to participate $\{24\}$}

Ethical approval was obtained prior enrolling patients from the clinical site. Ethics approval was granted for Mater Misericordiae University Hospital Institutional Review Board (IRB) on the 31/03/2021, reference: $1 / 378 / 2213$. Written, informed consent to participate will be obtained from all participants.

\section{Consent for publication $\{32\}$}

Model consent form and patient information leaflet can be provided on request

\section{Competing interests $\{28\}$}

The authors declare that they have no competing interests

\section{References}

1. Wong M, Sit A, Au T. Minimally invasive thoracic surgery: beyond surgical access. Journal of thoracic disease. 2018. doi:10.21037/jtd.2018.05.196.

2. Batchelor TJ, Rasburn NJ, Abdelnour-Berchtold E, et al. Guidelines for enhanced recovery after lung surgery: recommendations of the Enhanced Recovery After Surgery (ERAS ${ }^{\circledR}$ ) Society and the European Society of Thoracic Surgeons (ESTS). European journal of cardio-thoracic surgery. 2019. doi:10.1093/ejcts/ezy301.

3. Nagahiro I, Andou A, Aoe M, et al. Pulmonary function, postoperative pain, and serum cytokine level after lobectomy: a comparison of VATS and conventional procedure. The Annals of Thoracic Surgery. 2001. doi:10.1016/S0003-4975(01)02804-1. 
4. Li WW, Lee TW, Lam SS, Ng CS, Sihoe AD, Wan IY, Yim AP. Quality of life following lung cancer resection: video-assisted thoracic surgery vs thoracotomy. Chest. 2002. doi:10.1378/chest.122.2.584.

5. Schug SA, Bruce Julie. Risk stratification for the development of chronic postsurgical pain. Pain Reports. 2017. doi:10.1097/PR9.0000000000000627.

6. Finnerty DT, McMahon A, McNamara JR, Hartigan SD, Griffin M, Buggy DJ. Comparing erector spinae plane block with serratus anterior plane block for minimally invasive thoracic surgery: a randomised clinical trial. British journal of anaesthesia. 2020. doi:10.1016/j.bja.2020.06.020.

7. Karmakar MK. Thoracic Paravertebral Block. Anesthesiology. 2001. doi:10.1097/00000542200109000-00033.

8. Joshi GP, Bonnet F, Shah R, et al. A systematic review of randomized trials evaluating regional techniques for postthoracotomy analgesia. Anaesthesia analgesia. 2008. doi:10.1213/01.ane.0000333274.63501.ff.

9. Marhofer P, Feigl GC, Hopkins PH. Fascial plane block in regional anaesthesia: how problematic is simplification? British journal of anaesthesia. 2020. doi:10.1016/j.bja.2020.08.013.

10. Hill SE, Keller RA, Stafford-Smith M, et al. Efficacy of single-dose, multilevel paravertebral nerve blockade for analgesia after thoracoscopic procedures. Anesthesiology. 2006. doi:10.1097/00000542-200605000-00022.

11. Pawa A, Wojcikiewicz T, Barron A, et al. Paravertebral Blocks: Anatomical, Practical, and Future Concepts. Current Anesthesiology Reports. 2019; doi:10.1007/s40140-019-00328-x.

12. Shelley B. Macfie. Where now for thoracic paravertebral blockade? Anaesthesia. 2012. doi:10.1111/j.1365-2044.2012.07310.x.

13. Luyet C, Herrmann G, Ross S, et al. Ultrasound-guided thoracic paravertebral puncture and placement of catheter in human cadavers: where do catheters go? British journal of anaesthesia. 2011. doi:10.1093/bja/aeq309.

14. Cowie $B$, McGlade $D$, Ivanusic J, et al. Ultrasound-guided thoracic paravertebral blockade: a cadaveric study. Anaesthesia Analgesia. 2010. doi:10.1213/ANE.0b013e3181dd58b0.

15. Soni AK, Conacher ID, Waller DA, Hilton CJ. Video-assisted thoracoscopic placement of paravertebral catheters: A technique for postoperative analgesia for bilateral thoracoscopic surgery. $\mathrm{Br} \mathrm{J}$ Anaesth. 1994. doi:10.1093/bja/ael038.

16. Davies RG, Myles PS, Graham JM. A comparison of the analgesic efficacy and side-effects of paravertebral vs epidural blockade for thoracotomy-a systematic review and meta-analysis of randomized trials. British journal of anaesthesia. 2006. doi:10.1093/bja/ael020.

17. Myles PS, Myles DB, Galagher W, et al. Minimal Clinically Important Difference for Three Quality of Recovery Scales. Anesthesiology. 2016. doi:10.1097/ALN.0000000000001158.

18. Tulgar S, Kapakli MS, Senturk O, et al. Evaluation of ultrasound-guided erector spinae plane block for postoperative analgesia in laparoscopic cholecystectomy: a prospective, randomized, controlled clinical trial. J Clin Anesth. 2018. doi:10.1016/j.jclinane.2018.06.019. 
19. Gürkan Y, Aksu C, Kuş A, et al. Ultrasound guided erector spinae plane block reduces postoperative opioid consumption following breast surgery: a randomized controlled study. J Clin Anesth. 2018. doi:10.1016/j.jclinane.2018.06.033.

20. Krishna SN, Chauhan S, Bhoi D, et al. Bilateral erector spinae plane block for acute post-surgical pain in adult cardiac surgical patients: a randomized controlled trial. J Cardiothorac Vasc Anesth. 2019. doi:10.1053/j.jvca.2018.05.050.

21. Finnerty D, Ní Eochagáin A, Ahmed M, et al. A randomised trial of bilateral erector spinae plane block vs. no block for thoracolumbar decompressive spinal surgery. Anaesthesia. 2021. doi:10.1111/anae.15488.

22. Horth D, Sanh W, Moisiuk P, et al. Continuous erector spinae plane block versus intercostal nerve block in patients undergoing video-assisted thoracoscopic surgery: a pilot randomized controlled trial. Pilot Feasibility Study. 2021. doi:10.1186/s40814-021-00801-7.

23. Brunelli A, Thomas $C$, Dinesh P, Lumb A. Enhanced recovery pathway versus standard care in patients undergoing video-assisted thoracoscopic lobectomy. J Thorac Cardiovasc Surg. 2017;154:2084-90.

24. Piccioni F, Segat M, Falini S, et al. Enhanced recovery pathways in thoracic surgery from Italian VATS Group: peri-operative analgesia protocols. Journal Of Thoracic Disease. 2018. doi:10.21037/jtd.2017.12.86.

25. Ong CK, Lirk P, Seymour RA, Jenkins BJ. The efficacy of preemptive analgesia for acute postoperative pain management: a meta-analysis. Anesth Analg. 2005. doi:10.2147/JPR.S175169.

26. Dunn LK, Yerra S, Fang S, et al. Incidence and risk factors for chronic postoperative opioid use after major spine surgery: a cross-sectional study with longitudinal outcome. Anesth Analg. 2018. doi:10.1213/ANE.0000000000003338.

27. Hazapis $\mathrm{M}$, Walker $\mathrm{E}$, Rooms $\mathrm{M}$, et al. Measuring quality of recovery- 15 after day case surgery. $\mathrm{Br} \mathrm{J}$ Anaesth. 2016. doi:10.1093/bja/aev413.

\section{Figures}


Identify patients scheduled to undergo

MITS (VATS or RATS)

Patient meets inclusion criteria and has no exclusions

Patient gives informed

consent and is enrolled into

the study

Pre induction of GA maximum inspiratory volume measured bv bedside incentive soirometrv.

Induction of general anaesthesia
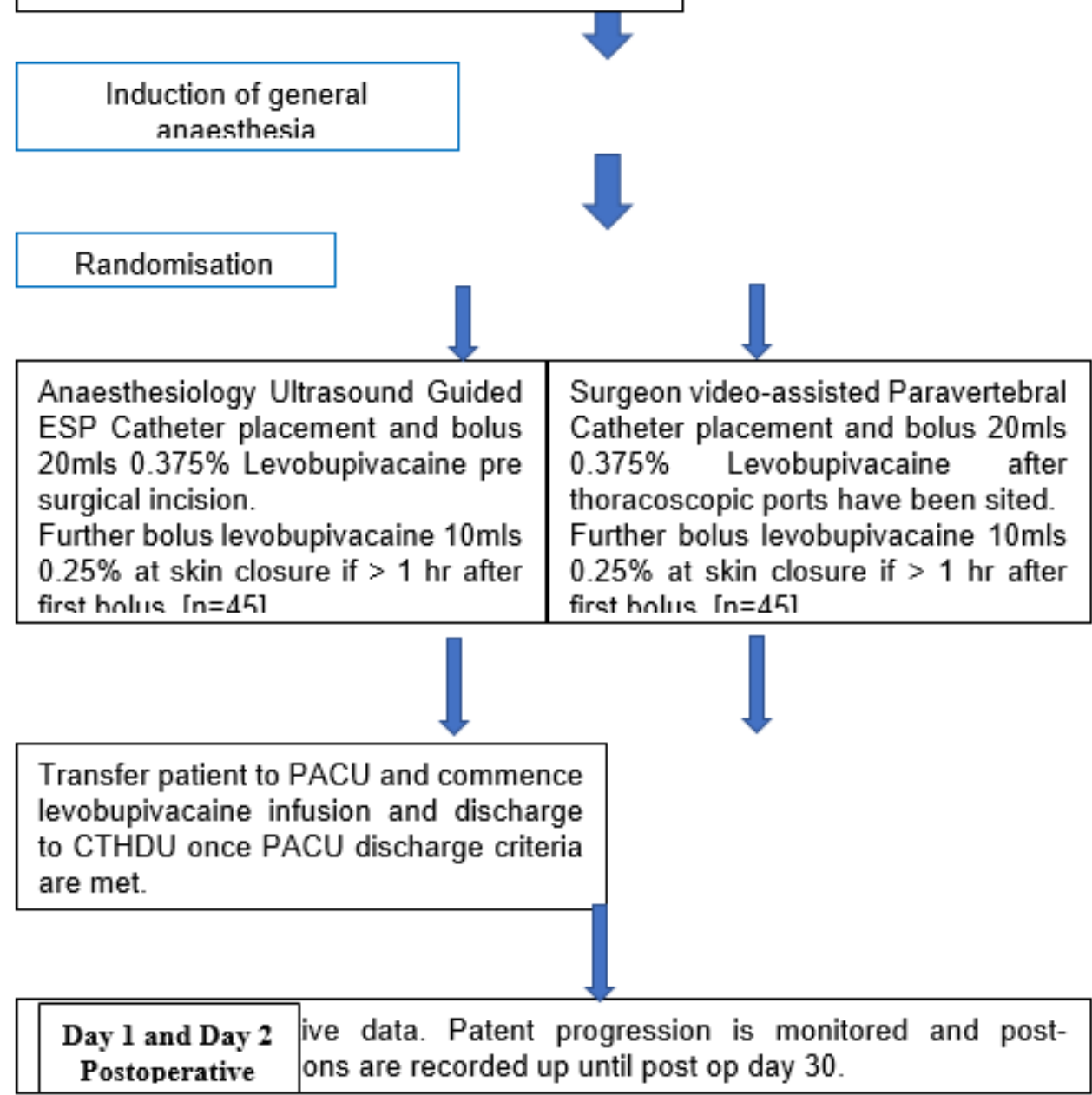

\section{Figure 1}

Study Flow Chart 


\section{Pre-surgical incision}

1. Paracetamol: 1 gram intravenously *dose adjustment required if patient's weight is $<50 \mathrm{~kg}$

2. NSAID (unless contraindicated):

nevlontenrofinn Enmo intraunnoush,

\section{Intra-operative period}

1. Intravenous (IV) opioid (fentanyl, morphine or oxycodone) at the discretion of the treating anaesthesiologist

2. Magnesium Sulphate 2 grams in $100 \mathrm{mls} 0.9 \%$ Normal saline IV over 30 mins.

3. Dexamethasone 4mg IV

4. Ondansetron $4 \mathrm{mg}$ IV

*avnid intra-nnerative usa of Remifentanil and Katamina

\section{Post Anaesthesia Care Unit (PACU)}

1. Commence $0.125 \% \mathrm{~L}$-Bupivacaine infusion at $10-15 \mathrm{ml} / \mathrm{hr}$ or $0.15 \% \mathrm{~L}$ Bupivacaine infusion at $8-12.5 \mathrm{ml} / \mathrm{hr}$ (Drug formulation dependant at clinical site)

2. As required Intravenous (IV) opioid (fentanyl, morphine or oxycodone) at the discretion of the treating anaesthesiologist

3. As required Clonidine $50-150 \mathrm{mcg}$ in $100 \mathrm{mls} 0.9 \%$ NS over 30 mins

4. As required anti-emetics (IV ondansetron $4 \mathrm{mg}$, Cyclizine $50 \mathrm{mg}$, Metoclopramide $10 \mathrm{mq} \mathrm{IV)}$

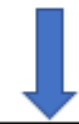

Post-operative (Cardiothoracic high dependence unit/ward)

1. PRN Oxycodone immediate release OxyNorm@ 5-10mg 2-4 hourly $\mathrm{PO}$ or Fentanyl PCA [20 mcg bolus at 6 min lock out, max $800 \mathrm{mcg}$ in four hours] at the discretion of the treating anaesthesiologist.

2. Paracetamol $1 \mathrm{~g}$ PO QDS for 48 hours

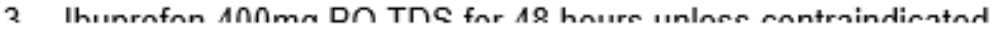

\section{Figure 2}

Analgesia and anti-emetic protocol 


\begin{tabular}{|c|c|c|c|c|c|c|c|}
\hline \multirow[b]{3}{*}{ TIMEPOINT } & \multicolumn{7}{|c|}{ STUDY PERIOD } \\
\hline & \multirow{2}{*}{$\begin{array}{c}\text { Enrolment } \\
\begin{array}{c}\text { Day before } \\
\text { surgery }\end{array}\end{array}$} & \multirow{2}{*}{$\begin{array}{c}\text { Allocation } \\
\begin{array}{c}\text { Day of } \\
\text { surgery }\end{array}\end{array}$} & \multicolumn{4}{|c|}{ Post-allocation } & \multirow{2}{*}{$\begin{array}{c}\text { Close-out } \\
\text { Length of } \\
\text { hospital } \\
\text { stay }\end{array}$} \\
\hline & & & $\begin{array}{l}\text { Post op } \\
\text { Day } 1\end{array}$ & $\begin{array}{c}\text { Post op } \\
\text { Day } 2\end{array}$ & $\begin{array}{c}\text { Post op } \\
\text { Day } 30\end{array}$ & $\begin{array}{c}\text { Post op } \\
\text { Day } 90\end{array}$ & \\
\hline \multicolumn{8}{|l|}{ ENROLMENT: } \\
\hline \multirow{3}{*}{$\begin{array}{l}\text { Eligibility screen } \\
\text { Informed consent } \\
\text { Baseline incentive } \\
\text { spirometer values }\end{array}$} & $X$ & & & & & & \\
\hline & $\mathrm{X}$ & & & & & & \\
\hline & $\mathrm{X}$ & & & & & & \\
\hline Allocation & & $X$ & & & & & \\
\hline \\
\hline \multicolumn{8}{|l|}{$\begin{array}{l}\text { Anaesthesiologist- } \\
\text { administered } \\
\text { ultrasound ESP } \\
\text { catheter }\end{array}$} \\
\hline \multicolumn{8}{|l|}{$\begin{array}{l}\text { Surgeon- } \\
\text { administered PVB } \\
\text { catheter }\end{array}$} \\
\hline \multicolumn{8}{|l|}{ ASSESSMENTS: } \\
\hline Qo5-15 score & & & $X$ & $X$ & & & \\
\hline \multicolumn{8}{|l|}{$\begin{array}{r}\text { CPSP using BPI \& } \\
\text { SF-15 } \\
\text { questionnaires }\end{array}$} \\
\hline $\begin{array}{l}\text { Post op incentive } \\
\text { spirometer values }\end{array}$ & & & $\mathrm{X}$ & $\mathrm{X}$ & & & \\
\hline Pain scores & & $X$ & $X$ & $\mathrm{X}$ & & & \\
\hline $\begin{array}{r}\text { Total opioid } \\
\text { consumption }\end{array}$ & & $X$ & $X$ & $\mathrm{X}$ & & & \\
\hline $\begin{array}{r}\text { Post-operative } \\
\text { complications } \\
\text { using } \mathrm{CCl}\end{array}$ & & & & & $X$ & & \\
\hline Length of stay & & & & & & & $x$ \\
\hline
\end{tabular}

Figure 3

Schedule of enrolment, interventions, and assessments. 